\title{
Broadband energy-efficient optical modulation by hybrid integration of silicon nanophotonics and organic electro-optic polymer
}

\author{
Xingyu Zhang*,a ${ }^{*}$ Amir Hosseini ${ }^{\mathrm{b}}$, Harish Subbaraman ${ }^{\mathrm{b}}$, Jingdong Luo ${ }^{\mathrm{c}}$, Alex K.-Y Jen ${ }^{\mathrm{c}}$, \\ Chi-jui Chunga ${ }^{\mathrm{a}}$, Hai Yan ${ }^{\mathrm{a}}$, Zeyu Pan ${ }^{\mathrm{a}}$, Robert L. Nelson ${ }^{\mathrm{d}}$, and Ray T. Chen*,a,b \\ ${ }^{a}$ University of Texas at Austin, 10100 Burnet Rd, MER 160, Austin, TX 78758, USA; \\ ${ }^{\mathrm{b}}$ Omega Optics, Inc., 8500 Shoal Creek Blvd, Austin, TX 78757, USA; \\ ${ }^{c}$ University of Washington, 302 Roberts Hall, Seattle, Washington 98195, USA; \\ ${ }^{d}$ Air Force Research Laboratory at Wright Patterson, Dayton, Ohio 45433, USA.
}

\begin{abstract}
Silicon-organic hybrid integrated devices have emerging applications ranging from high-speed optical interconnects to photonic electromagnetic-field sensors. Silicon slot photonic crystal waveguides (PCWs) filled with electro-optic (EO) polymers combine the slow-light effect in PCWs with the high polarizability of EO polymers, which promises the realization of high-performance optical modulators. In this paper, a broadband, power-efficient, low-dispersion, and compact optical modulator based on an EO polymer filled silicon slot PCW is presented. A small voltage-length product of $\mathrm{V}_{\pi} \times \mathrm{L}=0.282 \mathrm{~V} \times \mathrm{mm}$ is achieved, corresponding to an unprecedented record-high effective in-device EO coefficient $\left(\mathrm{r}_{33}\right)$ of $1230 \mathrm{pm} / \mathrm{V}$. Assisted by a backside gate voltage, the modulation response up to $50 \mathrm{GHz}$ is observed, with a $3-\mathrm{dB}$ bandwidth of $15 \mathrm{GHz}$, and the estimated energy consumption is $94.4 \mathrm{fJ} / \mathrm{bit}$ at $10 \mathrm{Gbit} / \mathrm{s}$. Furthermore, lattice-shifted PCWs are utilized to enhance the optical bandwidth by a factor of $\sim 10 \mathrm{X}$ over other modulators based on non-band-engineered PCWs and ring-resonators.
\end{abstract}

Keywords: integrated optics, microwave photonics, modulator, optical interconnects, photonic crystal, polymer, silicon photonics, slow light.

\section{INTRODUCTION}

The combination of silicon photonics and electro-optic (EO) polymers has enabled compact and highperformance hybrid integrated microwave photonic devices [1], such as modulators [2], interconnects [3] and sensors [4]. The large EO coefficient $\left(\mathrm{r}_{33}\right)$, ultrafast response time, very low dispersion, and spin-coating feature of EO polymers promise low-power consumption, ultra-high speed operation, and ease of fabrication [5-8]. Silicon photonics offers the potential of complementary metal-oxide-semiconductor (CMOS) compatible photonic integrated circuits $[9,10]$. Silicon photonic crystal waveguides (PCWs) $[11,12]$ exhibit slow-light effects which are beneficial for device miniaturization [13, 14]. Especially, EO polymer filled silicon slotted PCWs [15] further reduce the device size and enhance the device performance by combining the best of these two systems.

In this paper, we report the design, fabrication, and characterization results of a high-performance silicon-organic hybrid (SOH) PCW modulator with high modulation efficiency, broad RF bandwidth, low power consumption, and low optical dispersion. This paper is organized as follows. The design of a band-engineered PCW modulator and the principle of high-speed modulation are presented in the section on "principle and design". The section on "fabrication" describes the process of device fabrication. In the section on "results and discussion", we report the characterization results of our modulator, including $\mathrm{V}_{\pi} \times \mathrm{L}$, RF bandwidth, power consumption, and optical bandwidth. In the "conclusion" section, summary and future work are presented.

\section{DEVICE DESIGN}

\footnotetext{
*xzhang@utexas.edu; phone 1 512-471-4349; fax 1 512 471-8575

*raychen@uts.cc.utexas.edu; phone 1 512-471-7035; fax 1512 471-8575
} 


\section{a}

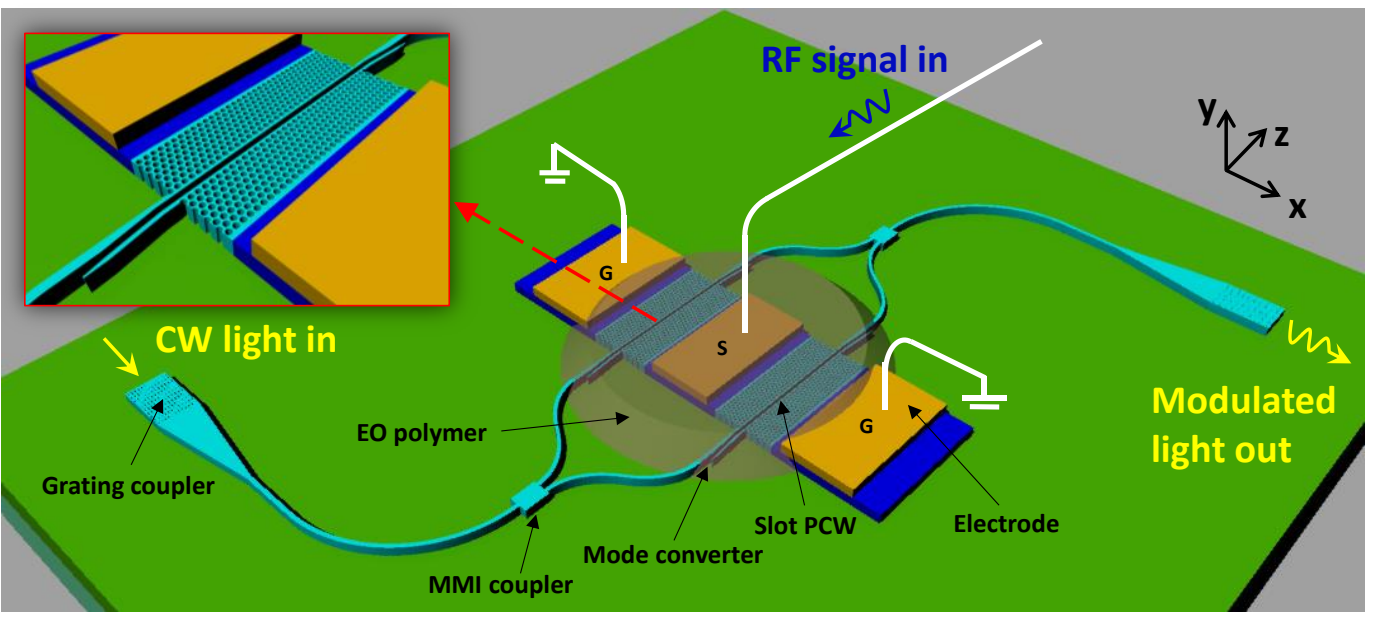

b

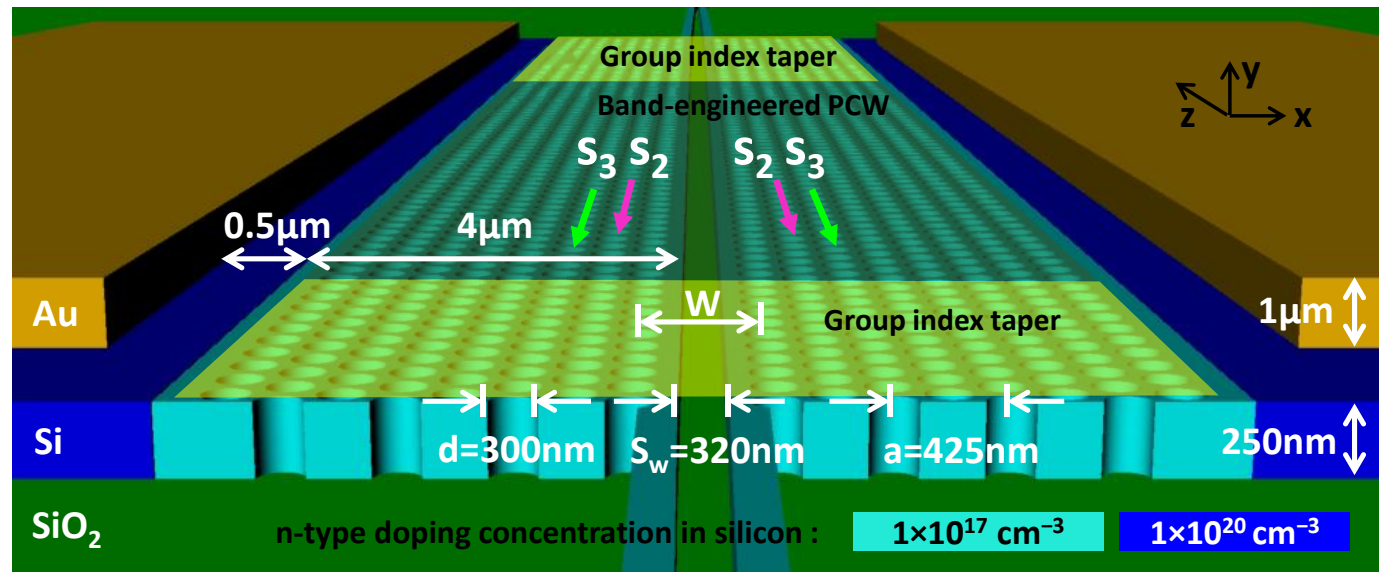

C

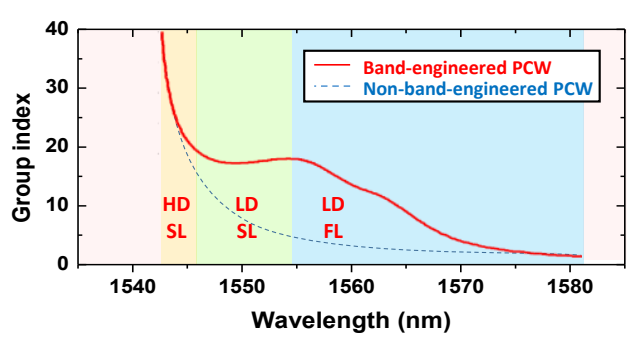

d

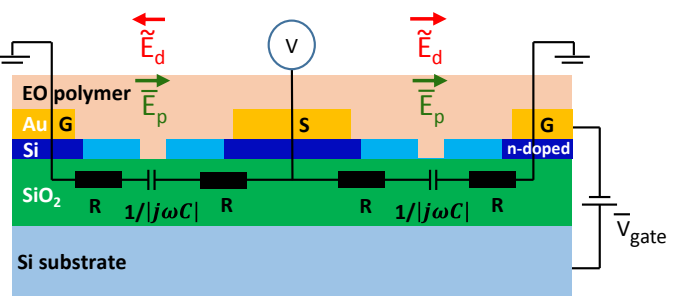

Fig. 1. An EO polymer filled silicon slot PCW MZI modulator designed on an SOI substrate. a, Three-dimensional schematic of the modulator. The inset shows the magnified image of the silicon slot PCW on one arm of the MZI. PCW: photonic crystal waveguide; MMI: Multi-mode interference; G: ground electrode; S: signal electrode. b, A tilted view of the slot PCW on one arm of the MZI, showing the cross-sectional device dimension, 2-level doping concentrations, group index taper region, and bandengineered PCW region. Note: the EO polymer is not shown here for better visualization. c. Simulation result of engineered group index in the slot PCW (red curve) as a function of wavelength, showing 8nm low-dispersion slow-light wavelength region (flat band nature of low-dispersion region highlighted in green). Also overlaid is a blue dashed curve representing the dispersive group index versus wavelength for non-band-engineered PCW for comparison. HD SL: high-dispersion slow-light; LD SL: lowdispersion slow-light; LD FL: low-dispersion fast-light. d, Equivalent electrical circuit of the MZI modulator in a push-pull configuration, with a constant gate voltage applied on the bottom silicon substrate. $\mathrm{E}_{\mathrm{d}}$ : driving field, $\mathrm{E}_{\mathrm{p}}$ : poling field, $\mathrm{V}_{\text {gate: }}$ gate voltage.

Our optical modulator is a symmetric Mach-Zehnder Interferometer (MZI), with slot photonic crystal waveguides (PCWs) incorporated in both the arms, as shown in Fig. 1a. We start with a silicon-on-insulator (SOI) substrate with 250nm-thick top silicon and $3 \mu \mathrm{m}$-thick buried oxide (BOX) layers. The slot and holes of the PCWs are filled with an EO polymer (SEO125 from Soluxra, LLC), which has a refractive index, $\mathrm{n}=1.63$ at $1550 \mathrm{~nm}$, and an exceptional 
combination of large EO coefficient ( $\mathrm{r}_{33}$ of $\sim 100 \mathrm{pm} / \mathrm{V}$ at $1550 \mathrm{~nm}$ ), low optical loss, synthetic scalability, as well as excellent photochemical stability. Its relatively high glass transition temperature of $150^{\circ} \mathrm{C}$ provides good temporal stability, and the EO coefficient of poled SEO125 is essentially unchanged under ambient conditions. The refractive index of the EO polymer can be changed by applying an electric field via the Pockels effect, and is given as $\Delta n=-1 / 2 r_{33} n^{3} V / S_{w}$, where $\Delta \mathrm{n}$ is the change in refractive index of the EO polymer, $\mathrm{V}$ is the applied voltage, $\mathrm{S}_{\mathrm{w}}$ is the slot width. The slot PCW has a hexagonal lattice of air holes with the lattice constant $a=425 \mathrm{~nm}$, hole diameter $\mathrm{d}=300 \mathrm{~nm}$, slot width $\mathrm{S}_{\mathrm{w}}=320 \mathrm{~nm}$, and center-to-center distance between two rows adjacent to the slot $\mathrm{W}=1.54(\sqrt{3}) \mathrm{a}$. The optimized slot width of $320 \mathrm{~nm}$ supports a confined optical mode, and also tremendously increases the EO polymer poling efficiency by suppressing the leakage current through the silicon/polymer interface during the poling process [16]. We also note that the poling-induced optical loss is reduced by this reduction of leakage current [17]. More importantly, different from typical slot widths of $100 \sim 120 \mathrm{~nm}$ in conventional slot waveguides $[15,18]$, widening the slot width to $320 \mathrm{~nm}$ reduces the slot capacitance, enabling the potential of higher RF bandwidth and lower power consumption, and relaxes the fabrication complexities. To address the issue of the narrow operational optical bandwidth of typical PCW modulators (less than $1 \mathrm{~nm}$ at the group index, $\mathrm{n}_{\mathrm{g}}>10$ ) [2, 19], lattices of the second and third rows of the PCW are shifted parallel to the slot with relative values of $S_{2}=-85 \mathrm{~nm}, S_{3}=85 \mathrm{~nm}$ (indicated by the arrows in Fig. 1b). As a result, a flat group index $\left(\mathrm{n}_{\mathrm{g}}\right)$ of $20.4( \pm 10 \%)$ over a wavelength range from $1546 \mathrm{~nm}$ to $1554 \mathrm{~nm}$ is achieved, as shown in Fig. 1c, enabling an optical spectrum range as wide as $8 \mathrm{~nm}$ for low-dispersion operation. In order to efficiently couple light from a strip waveguide into and out of the slot PCW, an adiabatic strip-to-slot mode converter is designed [20]. To make a smooth transition between the group indices from a slot waveguide ( $\mathrm{n}_{\mathrm{g}} \sim 3$ ) to a slot PCW ( $\left.\mathrm{n}_{\mathrm{g}} \sim 20.4\right)$, a group index taper consisting of 8 periods of non-lattice-shifted PCW is developed, in which W increases parabolically from $\mathrm{W}=1.45(\sqrt{3})$ a to $\mathrm{W}=1.54(\sqrt{3}) \mathrm{a}$ [21]. Sub-wavelength gratings (SWGs) are used to couple light into and out of the silicon strips [22]. Multi-mode interference (MMI) couplers are used for beam splitting/combining [21]. The PCW interaction length is chosen to be $300 \mu \mathrm{m}$ for $\mathrm{V}_{\pi}<1 \mathrm{~V}$ based on theoretical calculation using $L=\frac{1}{2 \sigma} \cdot \frac{n}{\Delta n} \cdot \frac{\lambda}{n_{g}}$ [23], where $\sigma=0.33$ is the confinement factor in the slot [24] calculated by the simulation, $\lambda=1550 \mathrm{~nm}$ is wavelength, and $\mathrm{n}_{\mathrm{g}}=20.4$.

Due to the short interaction length $(300 \mu \mathrm{m})$, the maximum modulation frequency of our modulator is not limited by the group velocity mismatch between RF and optical waves, which is usually the case in conventional modulation devices, necessitating the use of complex traveling wave electrode geometries. Instead, it is mainly limited by the time needed to charge the capacitor formed by the slot through the finite ohmic resistance across the silicon; therefore, our modulator can be driven by lumped electrodes [25-27]. The silicon PCW is selectively implanted by n-type dopant (Phosphorus ion, 31P+) with ion concentrations of $1 \times 10^{20} \mathrm{~cm}^{-3}$ and $1 \times 10^{17} \mathrm{~cm}^{-3}$ [28], as shown in Figs. 1a and b, so that the resistivity of silicon region is reduced to $9 \times 10^{-6} \Omega \cdot \mathrm{m}$ and $9 \times 10^{-4} \Omega \cdot \mathrm{m}$, respectively [29]. The purpose of using relatively lower concentration $\left(1 \times 10^{17} \mathrm{~cm}^{-3}\right)$ in the waveguide region is to avoid significant impurity-induced optical scattering loss $[30,31]$. The separation between the gold electrodes is $9.32 \mu \mathrm{m}$. Figure $1 \mathrm{~d}$ shows a simplified equivalent circuit of the modulator driven in a push-pull configuration, in which the slot can be represented by a capacitor C and the silicon PCW region by a resistor $\mathrm{R}$. As the modulation frequency increases, the percentage of electric potential dropped across the slot will decrease due to the reduced slot impedance $(1 / \mathrm{j} \omega \mathrm{C} \mid)$. The low resistivity of doped silicon can help increase the electric field inside the slot at high frequencies. Simulations by COMSOL Multiphysics show that over $90 \%$ of the electric potential is dropped across the slot at 10GHz. Both the optical field and the modulation RF field are concentrated in the $320 \mathrm{~nm}$-wide slot, enabling a large field interaction factor, and thus providing efficient modulation at high modulation frequency. Based on simulations performed using Lumerical Device software, the total resistance of the $300 \mu \mathrm{m}$-long silicon PCW is $189 \mathrm{Ohms}$, and the slot capacitance is as small as 39fF. Thus, the theoretical 3-dB modulation bandwidth of the MZI modulator is estimated to be $1 /(2 \pi R C)=22 \mathrm{GHz}$.

It was recently demonstrated that the RF bandwidth of modulators can be further improved by applying a constant gate voltage $\left(\mathrm{V}_{\text {gate }}\right)$ between the bottom silicon substrate and top silicon layer $[32,33]$ to make the top silicon layer sufficiently conductive. This technique avoids the need for heavy doping, so impurity-scattering optical loss can be minimized [34]. This method was used for conventional silicon slot waveguides to achieve modulation up to $42.7 \mathrm{GHz}$ [32] and low energy consumption of 320fJ/bit [33]. Here we apply a similar technique on our silicon PCW modulator, as shown in Fig. 1d. By applying a positive voltage on the backside silicon substrate (weakly doped, resistivity of $\sim 15 \Omega \cdot \mathrm{cm}$ ) across the $3 \mu \mathrm{m}$-thick BOX layer of our device, the energy bands in the $\mathrm{n}$-type top silicon are bent, and thus more electrons accumulate at the interface between the silicon PCW and the BOX layer. Since the resistivity of the silicon region is inversely proportional to the density and mobility of majority free carriers, the resistivity of the silicon PCW region can be reduced, leading to an enhanced RF bandwidth and a reduced power consumption of the modulator. 


\section{FABRICATION}

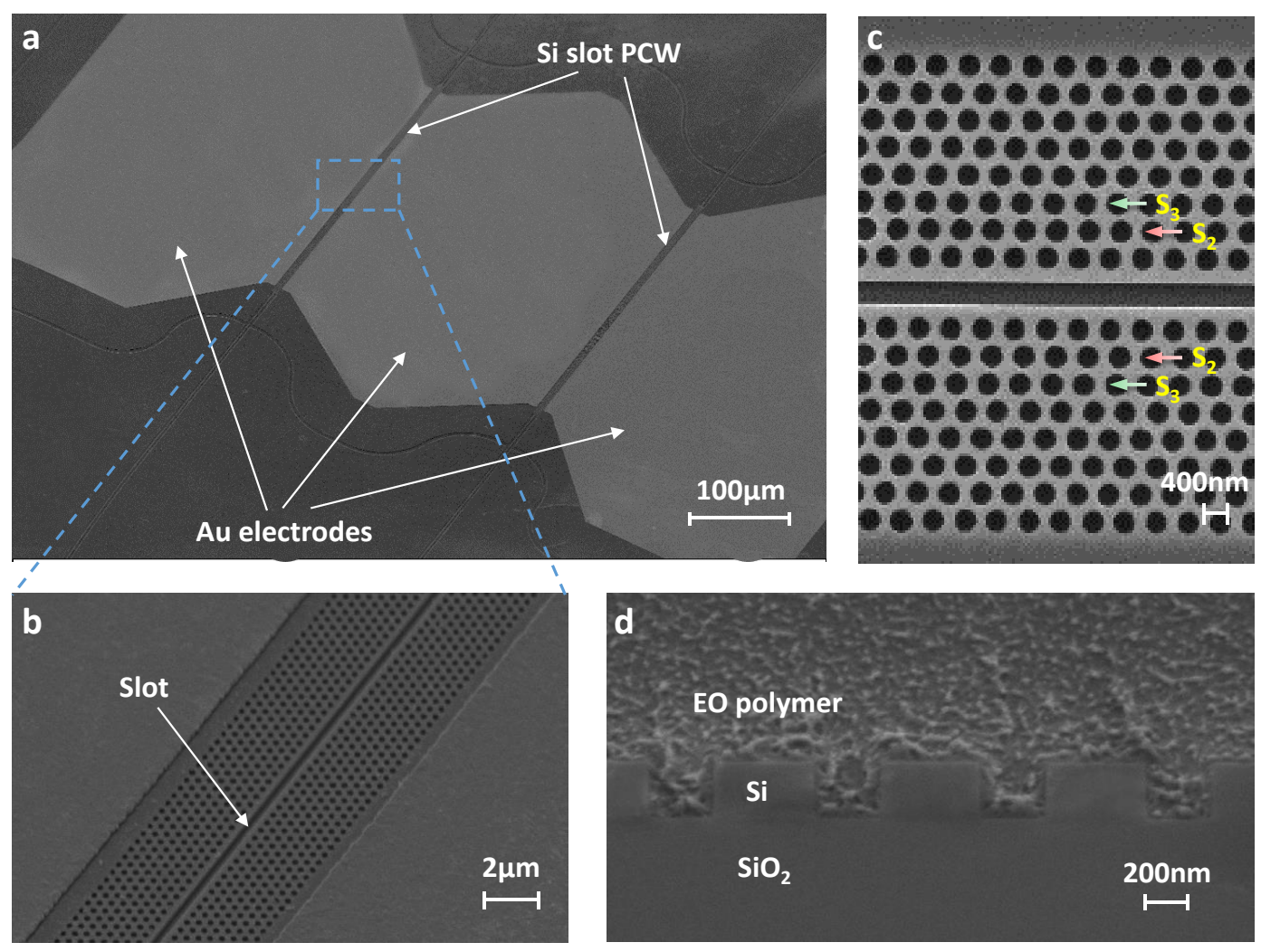

Fig. 2. SEM images of the fabricated device. a, A tilted view of the symmetric MZI modulator with silicon slot PCWs in both arms. b, A magnified image of the silicon slot PCW in one arm inside the gap of electrodes. c, A top view of the slot PCW, with arrows indicating the shifted lattices on the second and third rows. $S_{2}=-85 \mathrm{~nm}$, and $S_{3}=85 \mathrm{~nm}$. d, A cross-sectional view of the photonic crystal structure filled with EO polymer.

The fabrication procedure starts with an SOI wafer. The silicon slot PCW is patterned by electron-beam lithography and reactive ion etching (RIE). Then, the silicon slot PCW is first implanted with $31 \mathrm{P}+$ at energy of $92 \mathrm{keV}$ and dose of $1.05 \times 10^{12} / \mathrm{cm}^{2}$ to reach an ion concentration of $1 \times 10^{17} \mathrm{~cm}^{-3}$. Next, the device is patterned by photolithography and selectively implanted with $31 \mathrm{P}+$ at energy of $92 \mathrm{keV}$ and dose of $1.05 \times 10^{15} / \mathrm{cm}^{2}$ to reach an ion concentration of $1 \times 10^{20} \mathrm{~cm}^{-3}$ in the region which will connect the gold electrodes in order to form ohmic contacts. A rapid thermal annealing at $1000^{\circ} \mathrm{C}$ for $10 \mathrm{~min}$ in a flowing nitrogen environment is followed to annihilate the induced defects and activate the implanted ions, which also improves the optical performance of the ion-implanted waveguides. Next, $1 \mu \mathrm{m}$-thick gold electrodes with $5 \mathrm{~nm}$-thick chromium adhesion layers are patterned using photolithography, electron-beam evaporation, and lift-off. Figures $2 \mathrm{a}$ and $\mathrm{b}$ show the SEM images of the fabricated device in a tilted view. Figure 2c shows a top view of the fabricated slot PCW, with arrows indicating the shifted lattices. Next, the EO polymer is formulated, and then covered over the PCW and dispersed into the holes and slot by spin coating. Figure $2 \mathrm{~d}$ shows the cross section after EO polymer filling.

Finally, to activate the EO effect, a poling process is performed [35-37]. The device is heated up on a hot plate to the EO polymer glass transition temperature of $150^{\circ} \mathrm{C}$ in a nitrogen atmosphere, and a constant poling electric field of $110 \mathrm{~V} / \mu \mathrm{m}$ is applied across the EO polymer inside the slot in a push-pull configuration, as shown in Fig. 1d. The randomly oriented chromophore dipoles inside the polymer matrix are then free to rotate and align in the direction of poling electric field. Next the temperature is quickly decreased to room temperature while the constant electric field is still applied, and eventually the chromophores are locked in a uniform direction to form a noncentrosymmetric structure. During this poling process, the leakage current is monitored and it remains below $0.53 \mathrm{nA}$, corresponding to a low leakage current density of $5.5 \mathrm{~A} / \mathrm{m}^{2}$. This is comparable to the typical leakage current density of $1-10 \mathrm{~A} / \mathrm{m}^{2}$ measured in a thin film configuration, indicating a high poling efficiency [38]. 


\section{CHARACTERIZATION}
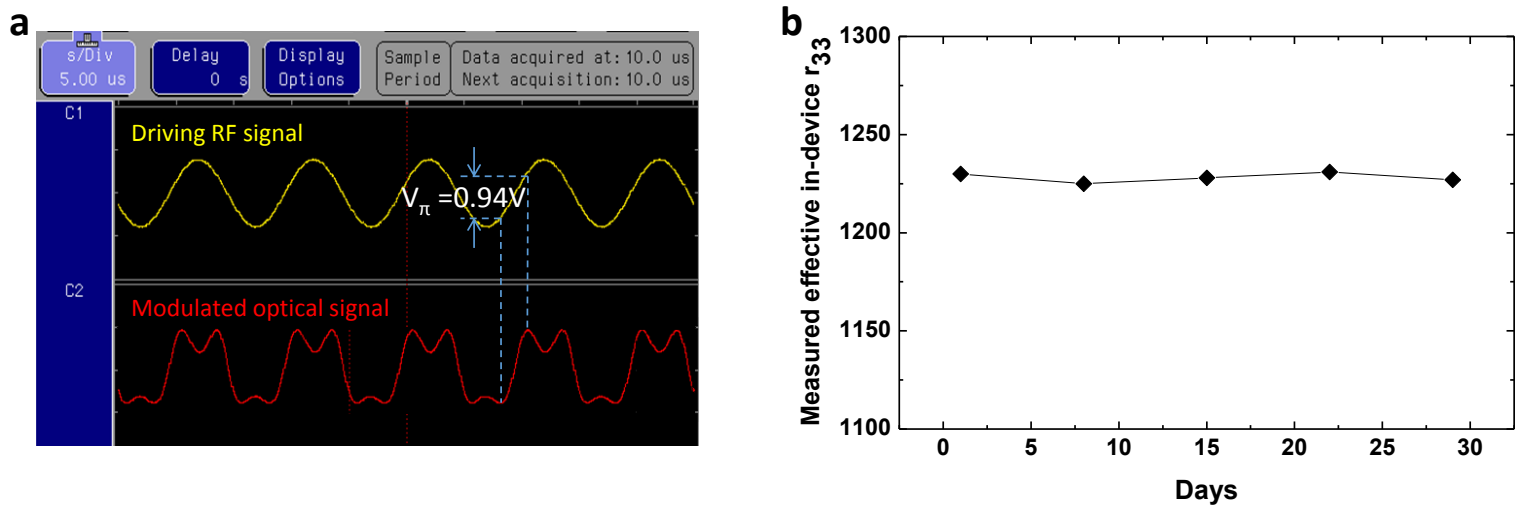

Fig. 3. Device characterization at low frequency. a, Transfer function at $100 \mathrm{kHz}$. The $\mathrm{V}_{\pi}$ is measured to be $0.94 \mathrm{~V}$ from overmodulation. b, Measured effective in-device $\mathrm{r}_{33}$ as a function of time in days, indicating the long-term stability of the modulator.

A low-frequency modulation test is first performed on the device to measure the voltage-length product, $\mathrm{V}_{\pi} \times \mathrm{L}$, which is a figure of merit (FOM) for optical modulators. TE-polarized light from a tunable laser source (1550nm) is coupled into and out of the device utilizing an in-house built grating coupler setup [22]. An RF signal from a function generator is applied onto the electrodes in a push-pull configuration, as shown in Fig. 1d. The modulator is biased at the $3 \mathrm{~dB}$ point and driven by a sinusoidal $\mathrm{RF}$ wave with a peak-to-peak voltage of $\mathrm{V}_{\mathrm{d}}=1.5 \mathrm{~V}$ at $100 \mathrm{KHz}$. The modulated output optical signal is detected using an amplified avalanche photodetector and a digital oscilloscope setup. As shown in Fig. 3a, over-modulation is observed on the output optical waveform, and the $\mathrm{V}_{\pi}$ of the modulator is measured to be $0.94 \mathrm{~V}$. Thus, the FOM of the modulator achieved is $\mathrm{V}_{\pi} \times \mathrm{L}=0.94 \mathrm{~V} \times 300 \mu \mathrm{m}=0.282 \mathrm{~V} \times \mathrm{mm}$. The effective in-device $\mathrm{r}_{33}$ is then calculated to be [4]

$$
r_{33, e f f}=\frac{\lambda S_{w}}{n^{3} V_{\pi} \sigma L}=1230 \mathrm{pm} / \mathrm{V}
$$

where $\lambda=1550 \mathrm{~nm}, \mathrm{~S}_{\mathrm{w}}=320 \mathrm{~nm}, \mathrm{n}=1.63, \mathrm{~L}=300 \mu \mathrm{m}$, and $\sigma=0.33$, which is the highest ever in-device $\mathrm{r}_{33}$ ever recorded. Such a high $r_{33}$ value originates from the combined effects of a large bulk $r_{33}$ of the EO polymer material, an improved poling efficiency achieved via widening the slot width $(320 \mathrm{~nm})$, the slow-light enhancement in the silicon PCW, as well as the increased percentage of voltage drop across the slot due to silicon doping. Discounting the slow-light effect, the actual in-device $r_{33}$ is estimated to be as high as $98 \mathrm{pm} / \mathrm{V}$ [39-41]. In addition, to verify the long-term stability of the device, the same test is repeated in the same conditions over the duration of a month, and the measured effective in-device $r_{33}$ as a function of time in days is shown in Fig. 3b. It can be seen that no severe degradation of device performance is observed after a month, due to the improved stability of the EO polymer material.

The RF bandwidth is measured in a small signal modulation test. RF driving signal is provided by a vector network analyzer (VNA) and applied onto the electrodes of the modulator via a ground-signal-ground (GSG) picoprobe. The modulated optical signal is amplified by an erbium doped fiber amplifier (EDFA) and received by a high-speed photodetector, and then the received power is measured using a microwave spectrum analyzer (MSA). The measured EO response of the device as a function of modulation frequency is shown in Fig. 4a, from which a 3-dB bandwidth of $11 \mathrm{GHz}$ is measured. Note that the upper frequency of this measurement is limited by the upper limit of our MSA, which is $26 \mathrm{GHz}$.

Next, in order to overcome this measurement limit and demonstrate the modulation response at frequencies over $26 \mathrm{GHz}$, we perform another measurement using a sideband detection technique [42-45]. The optical output of the modulator is directly connected to the optical spectrum analyzer (OSA), and the transmission spectrum of the modulator is measured. When the modulator is driven by a high frequency RF signal, two sidebands appear in the transmission spectrum, equally spaced around the main peak [42-46]. Figure $4 \mathrm{~b}$ shows overlaid transmission spectra of the optical modulator driven at $10 \mathrm{GHz}, 20 \mathrm{GHz}, 30 \mathrm{GHz}$ and $40 \mathrm{GHz}$. At higher modulation frequencies, the power of the sidebands becomes lower due to the combined effects of decreased electric potential drop across the slot, reduced output power of the RF source, and increased RF loss on the feeding cable and the probe. Since the power of the main peak and first sideband is proportional to the square of the zero-order and first-order Bessel function of the first kind $\left(\mathrm{J}_{\mathrm{i}}, \mathrm{i}=0,1\right)$ as a function of phase modulation index $(\eta)$ which represents the achieved phase shift (unit: 
radians), by measuring the ratio of the main peak power and sideband power $\left(\mathrm{J}_{0}^{2}(\eta) / \mathrm{J}_{0}^{2}(\eta) \approx(2 / \eta)^{2}\right)$, the phase modulation index $(\eta)$ can be extracted [32, 44-46]. The obtained modulation index as a function of modulation frequency is plotted as the red curve shown in Fig. 4c. Sideband signals are observed above the noise floor until the modulation frequency is over $43 \mathrm{GHz}$.
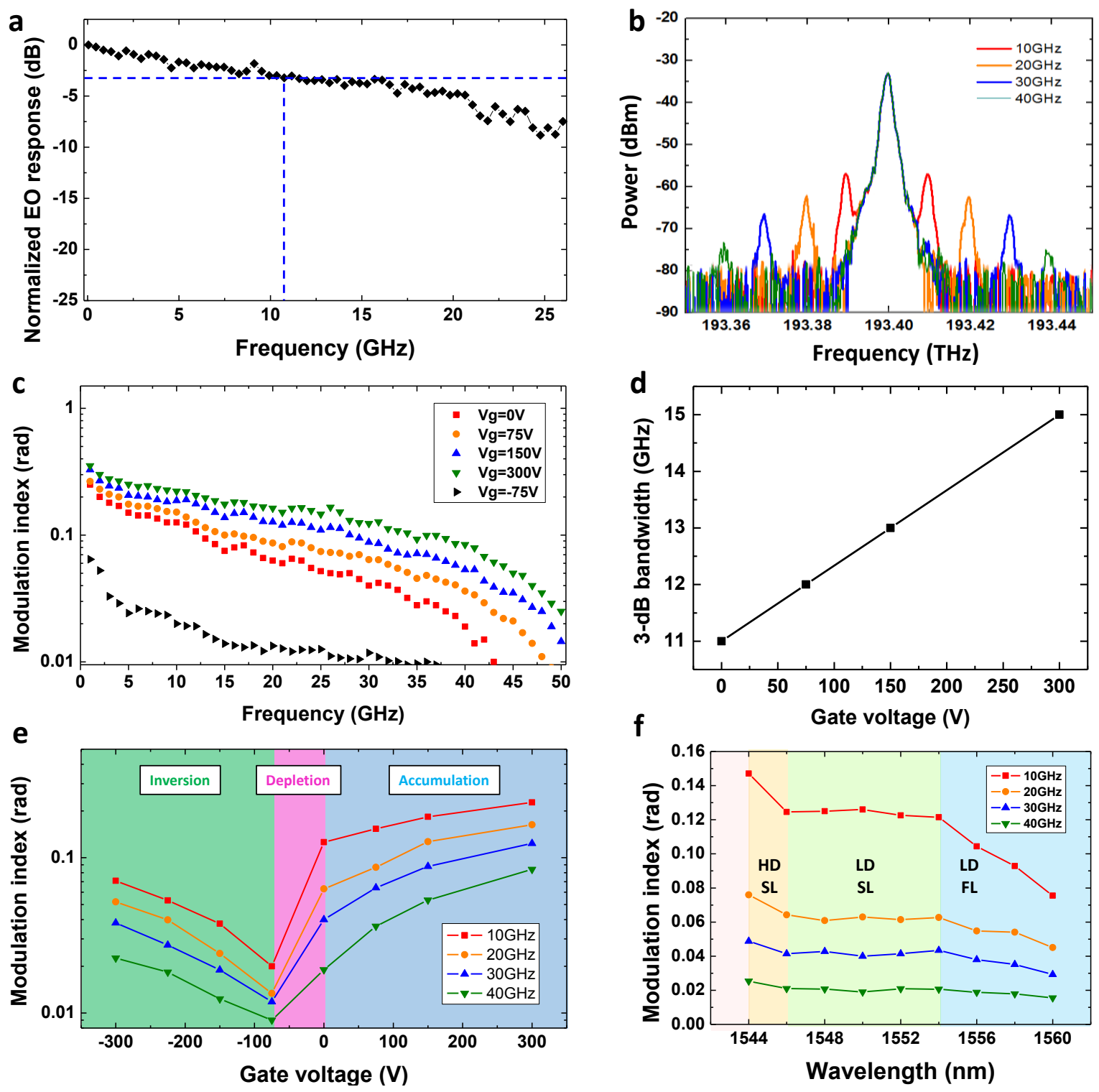

Fig. 4 Device characterization at high frequency. a, Measured normalized EO response of the modulator as a function of modulation frequency in a small-signal modulation test. The 3-dB bandwidth is measured to be $11 \mathrm{GHz}$. b, Measured optical transmission spectra of the modulator operating at $10 \mathrm{GHz}, 20 \mathrm{GHz}, 30 \mathrm{GHz}$ and $40 \mathrm{GHz}$. c, Measured modulation index as a function of frequency, under different backside gate voltages. d, Increased 3-dB RF bandwidth as the positive gate voltage increases. e, Measured modulation index as a function of $\mathrm{V}_{\text {gate }}$ at different modulation frequencies, overlaid with the states of accumulation, depletion and inversion. f, Measured modulation index over a range of optical wavelengths. The modulation index is nearly constant over a low-dispersion slow-light region of $8 \mathrm{~nm}$.

Next, to further increase the RF bandwidth of the modulator, a positive gate voltage $\left(\mathrm{V}_{\text {gate }}\right)$ from a high-voltage supply is applied to the bottom silicon substrate across the BOX layer of our device [32, 33], as shown in Fig. 1d. The positive $V_{\text {gate }}$ is varied and the corresponding modulation index is measured. As shown in Fig. 4c, as the positive $V_{\text {gate }}$ increases, the measured modulation index at each frequency increases and the whole curve becomes flatter, due to the 
increased electron accumulation at the interface of the silicon PCW and the BOX [32,33]. When the $\mathrm{V}_{\text {gate }}$ is increased over $150 \mathrm{~V}$, the sideband power starts to appear above the noise level in the transmission spectrum at $50 \mathrm{GHz}$, indicating the achievable frequency response of our modulator at up to 50GHz. This measured maximum modulation frequency is limited by the available frequency range of our RF source. Figure $4 \mathrm{~d}$ shows the 3 -dB bandwidth of the modulator as a function of positive $\mathrm{V}_{\text {gate }}$, and it can be seen that under the $\mathrm{V}_{\text {gate }}$ of $300 \mathrm{~V}$, the 3-dB bandwidth is increased to $15 \mathrm{GHz}$. Note the breakdown electric field of the silicon dioxide is about $0.5 \mathrm{GV} / \mathrm{m}$ [47], corresponding to a voltage of $1500 \mathrm{~V}$ that the $3 \mu \mathrm{m}$-thick BOX layer can withstand.

In order to further investigate the device performance under $\mathrm{V}_{\text {gate }}$, a negative $\mathrm{V}_{\text {gate }}$ is applied, and the modulation index is measured and plotted in Fig. 4e. It can be seen that with the magnitude of negative voltage slightly increased, the measured modulation index decreases due to the depletion of electrons. At a $V_{\text {gate }}$ of around $-75 \mathrm{~V}$, the free electrons are almost completely depleted, so the modulation index becomes the smallest, which is also shown by the black curve in Fig. 4c. When the magnitude of the negative voltage further increases, modulation index starts to increase because "inversion" state occurs in which holes are accumulated in the top silicon PCW layer. This interesting phenomenon is quite similar to the well-known Metal-Oxide-Semiconductor (MOS) capacitor structure [48].

What is more, a small switching voltage and low power consumption is achieved under a high positive $\mathrm{V}_{\text {gate }}$. For example, the measured modulation index is $\eta=0.23$ at $10 \mathrm{GHz}$ under $\mathrm{V}_{\text {gate }}=300 \mathrm{~V}$, and correspondingly, the required switching voltage is calculated to be $V_{\pi}=\pi / \eta \times V_{d}=2.2 \mathrm{~V}$ at $10 \mathrm{GHz}$, where $\mathrm{V}_{\mathrm{d}}=0.16 \mathrm{~V}$ is the $\mathrm{RF}$ driving voltage calculated from the output power of RF source. Since our modulator is a lumped device without termination, the power consumption is then dominated by the capacitive load of the slot. The RF power consumption for $100 \%$ modulation depth is $2 \pi f \times\left(1 / 2 C V_{\pi}^{2}\right) \times 2=24 \mathrm{~mW}$ at modulation frequency of $\mathrm{f}=10 \mathrm{GHz}$, where $\mathrm{C}=39 \mathrm{fF}$ is the slot capacitance calculated by the simulation, $\mathrm{V}_{\pi}=2.2 \mathrm{~V}$ is used as the driving voltage to achieve a maximum extinction ratio, and a factor of 2 is added due to the push-pull configuration. In addition, we make an estimation of energy consumption per bit for our device [49]. If our modulator is driven by PRBS signals with the same power level, we estimate the energy consumption per bit for our modulator at the bit rate of $10 \mathrm{Gbit} / \mathrm{s}$ as $W_{b i t}=\frac{1}{4} C V_{\pi}^{2} \times 2=94.4 \mathrm{fJ} / \mathrm{bit}[26,33,45,49$, 50]. Note that, in actual high-speed digital modulations, the driving voltage can be smaller than $\mathrm{V}_{\pi}$, in which case a decently clear eye diagram, a high enough extinction ratio and acceptable bit error rate (BER) can be still achieved using lower energy $[14,19,33,51,52]$. Though, we still use $\mathrm{V}_{\pi}$ as driving voltage (i.e.100\% modulation depth) in our estimation, because this can compensate the actual voltage drop caused by experimental imperfections such as reflections, drift, RF loss, etc., and allows for a reasonable estimated value of energy consumption. This very low estimated energy consumption is due to both a significantly reduced $V_{\pi}$ and the very small capacitance achieved by widening the slot. Note that, although the applied $\mathrm{V}_{\text {gate }}$ is high, the power consumption on the backside gate is negligible $(<30 \mathrm{pW})$ due to the highly insulating BOX layer.

Finally, to demonstrate the wide optical bandwidth of this PCW modulator, the wavelength of the laser input is tuned from $1544 \mathrm{~nm}$ to $1560 \mathrm{~nm}$, while $\mathrm{V}_{\text {gate }}$ is set to be zero and all other testing conditions are kept the same. Over this spectrum range, the modulation index is measured at $10 \mathrm{GHz}, 20 \mathrm{GHz}, 30 \mathrm{GHz}$, and $40 \mathrm{GHz}$, and the results are plotted in Fig. 4f. It can be seen that at each modulation frequency, the curve of the measured modulation index looks flat from $1546 \mathrm{~nm}$ to $1554 \mathrm{~nm}$, with a small variation of $\pm 3.5 \%$. This is because the modulation index is proportional to the $n_{\mathrm{g}}\left(\eta \sim 1 / \mathrm{V}_{\pi}\right.$ and $\mathrm{V}_{\pi} \sim \mathrm{B} \times \lambda / \mathrm{n}_{\mathrm{g}}$, where $\mathrm{B}$ is a constant) [53], and $\mathrm{n}_{\mathrm{g}}$ has been engineered to be almost constant in this low-dispersion slow-light wavelength region, as shown in Fig. 1c. This 8nm-wide low-dispersion spectrum range is useful for some applications such as wavelength division multiplexing (WDM), and also makes our modulator insensitive to variations of wavelength and temperature, which is much better than non-band-engineered PCWs [2, 19] and ring resonators [54-57]. In Fig. 4f, the largest modulation index is achieved in the high-dispersion slow-light region (from $1543.5 \mathrm{~nm}$ to $1546 \mathrm{~nm}$ ), because of the largest $\mathrm{n}_{\mathrm{g}}$ in this region. As the wavelength increases over $1554 \mathrm{~nm}$, the measured modulation index decreases due to the decreasing value of $n_{\mathrm{g}}$.

\section{DISCUSSIONS}

In recent years, quite a few research groups reported impressive work on analog/digital optical modulators based on similar structures such as silicon PCW MZI [14] and EO polymer filled silicon slot waveguide MZI [33], while our EO polymer filled silicon slot PCW MZI modulator combines the benefits from both the slow-light PCW [14] and 
the silicon-organic hybrid (SOH) structure [33]. In Reference [14], Nguyen, et al, demonstrated a silicon MachZehnder modulators with $90 \mu \mathrm{m}$-long lattice-shifted photonic crystal waveguides with $n_{g}=20 \sim 30$. By utilizing the plasma dispersion effect on $\mathrm{p}-\mathrm{n}$ diode, digital modulation at a data rate of $40 \mathrm{Gbit} / \mathrm{s}$, optical bandwidth of $12.5 \mathrm{~nm}$ were experimentally demonstrated. However, only a peak-to-peak driving voltage, $\mathrm{V}_{\mathrm{pp}}=5.3 \mathrm{~V}$ (instead of $\mathrm{V}_{\pi}$ ), is reported in this reference, so we theoretically estimate the $\mathrm{V}_{\pi}$ of the modulator $[23,53,58]$ to be $V_{\pi}=\pi /\left(\frac{2 \pi}{\lambda} \Delta n_{\text {eff }} n_{g} L\right)$ $=\pi /\left(\frac{2 \pi}{1550 \mathrm{~nm}} \cdot 1.6 \times 10^{-5} \cdot 30 \cdot 90 \mu \mathrm{m}\right)=18 \mathrm{~V}$ for apples-to-apples comparison. This large $\mathrm{V}_{\pi}$ value leads to a large $\mathrm{V}_{\pi} \times \mathrm{L}$ product of $1.62 \mathrm{~V} \times \mathrm{mm}$ at $10 \mathrm{Gbit} / \mathrm{s}$. In reference [33], Palmer, et al, reported a MZI modulator in which EO polymer is filled into a $1.5 \mathrm{~mm}$-long slot waveguide with slot width of $80 \mathrm{~nm}$. This modulator was demonstrated with in-device $r_{33}$ of $15 \mathrm{pm} / \mathrm{V}$, energy consumption of $320 \mathrm{fJ} / \mathrm{bit}$ at $10 \mathrm{Gbit} / \mathrm{s}$ digital modulation, and $\mathrm{V}_{\pi}=2.5 \mathrm{~V}$ which corresponds to a $\mathrm{V}_{\pi} \times \mathrm{L}=3.75 \mathrm{~V} \times \mathrm{mm}$. In a recent reference published by the same group [59], Alloatti, et al, demonstrated a MZI modulator with $500 \mu \mathrm{m}$-long $120 \mathrm{~nm}$-wide-slot waveguide filled with EO polymer, in which they reported a $3 \mathrm{~dB}$ bandwidth of $100 \mathrm{GHz}$, but a relatively higher $\mathrm{V}_{\pi} \times \mathrm{L}=11 \mathrm{~V} \times \mathrm{mm}$. In addition, recently, a rather specialized SOH modulator based on plasmonic waveguide has been demonstrated by Melikyan, et al, with bit rate of $40 \mathrm{Gbit} / \mathrm{s}$, energy consumption of $60 \mathrm{fJ} / \mathrm{bit}$, optical bandwidth of $120 \mathrm{~nm}$ [45]. Considering the reported $\mathrm{V}_{\pi} \times \mathrm{L}=1.3 \mathrm{Vmm}$ and device length of $29 \mathrm{um}$, its value of $\mathrm{V}_{\pi}$ is as large as $45 \mathrm{~V}$, which is prohibitive for many applications. With the continuous efforts and positive contributions made by researchers in this area, more research progress with improved device performance is expected to be reported in the future.

In our future work, the $\mathrm{V}_{\pi} \times \mathrm{L}$ product can also be further reduced by using more efficient organic $\mathrm{EO}$ materials, such as supramolecular organic EO glasses and binary EO polymers exhibiting intrinsic Pockels coefficients greater than $300 \mathrm{pm} / \mathrm{V}$ [60]. And also, the poling efficiency can be improved by using pyroelectric poling technique [61]. A transmission line can be designed to drive the modulator as a traveling wave device, in order to achieve modulation frequency over $100 \mathrm{GHz}[59,62]$. Recently, the $\mathrm{SOH}$ slot waveguide structures have been developed for digital modulations more advanced modulation formats such as QPSK and 16QAM with $>1 \mathrm{~Tb} / \mathrm{s}$ [63, 64], thus, our future work will also include driving our modulator using high-speed digital signals $[14,19,52]$ and also using this modulator for advanced modulation formats and coherent modulation scheme [63, 64]. In addition, potential stability of the modulator, a common issue for almost all polymer based devices, can be further improved by developing new EO polymers with higher glass transition temperatures and crosslinking chemistry, and by hermetically sealing the EO polymer and removing oxygen in the device packaging [65]. More detailed studies of EO polymer thermal stability indicate that operation up to $150^{\circ} \mathrm{C}$ results in a change of $\mathrm{EO}$ coefficients $<10 \%[66,67]$.

\section{CONCLUSION}

In summary, we demonstrate a broadband, low-power, low-dispersion and compact optical modulator based on a silicon slot PCW filled with EO polymer. Benefiting from the combined enhancement provided by the slow-light effect and high- $\mathrm{r}_{33}$ polymer, the voltage-length product of the modulator is measured to be as small as $\mathrm{V}_{\pi} \times \mathrm{L}=0.282 \mathrm{~V} \times \mathrm{mm}$, corresponding to a record-high effective in-device $\mathrm{r}_{33}$ of $1230 \mathrm{pm} / \mathrm{V}$ ever demonstrated. The silicon PCW is selectively doped to reduce the RC time delay and to achieve high-speed modulation. A backside gate technique is applied to our silicon PCW device to enhance device performance. Assisted by the backside gate voltage of $300 \mathrm{~V}$, the $3-\mathrm{dB}$ bandwidth of the modulator is demonstrated to be $15 \mathrm{GHz}$, and a modulation response up to $50 \mathrm{GHz}$ is observed. To the best of our knowledge, this is the first experimental demonstration of a 50GHz SOH PCW MZI modulator, although a 50GHz silicon /polymer hybrid slot MZI modulators [33], a 40GHz silicon /polymer hybrid PCW band-edge-shift modulator [42], and a 40Gbit/s monolithic silicon PCW MZI modulators [14] were previously demonstrated. In addition, the power consumption of the modulator is measured to be $24 \mathrm{~mW}$ at $10 \mathrm{GHz}$, and the estimated value of energy consumption per bit for a potential digital modulation is approximately $94.4 \mathrm{fJ} / \mathrm{bit}$ at $10 \mathrm{Gbit} / \mathrm{s}$ based on measured $\mathrm{V}_{\pi}$ at $10 \mathrm{GHz}[26,33,45,49,50]$. By using the band-engineered PCWs, the modulator is demonstrated to have a low-dispersion optical spectrum range as wide as $8 \mathrm{~nm}$, which is a factor of $\sim 10 \mathrm{X}$ better than other modulators based on non-band-engineered PCWs [2,19] and ring resonators [54] which have narrow operating optical bandwidth of $<1 \mathrm{~nm}$. 


\section{ACKNOWLEDGEMENT}

The authors would like to acknowledge the Air Force Research Laboratory (AFRL) for supporting this work under the Small Business Technology Transfer Research (STTR) program (Grant No. FA650-12-M-5131) monitored by Drs. Rob Nelson and Charles Lee.

\section{REFERENCES}

[1] C. Koos, P. Vorreau, T. Vallaitis, P. Dumon, W. Bogaerts, R. Baets, B. Esembeson, I. Biaggio, T. Michinobu, and F. Diederich, "All-optical high-speed signal processing with silicon-organic hybrid slot waveguides," Nature Photonics, 3(4), 216-219 (2009).

[2] J. H. Wülbern, J. Hampe, A. Petrov, M. Eich, J. Luo, A. K.-Y. Jen, A. Di Falco, T. F. Krauss, and J. Bruns, "Electro-optic modulation in slotted resonant photonic crystal heterostructures," Applied Physics Letters, 94(24), 241107 (2009).

[3] X. Zhang, A. Hosseini, X. Lin, H. Subbaraman, and R. T. Chen, "Polymer-based Hybrid Integrated Photonic Devices for Silicon On-chip Modulation and Board-level Optical Interconnects," IEEE Journal of Selected Topics in Quantum Electronics, 19(6), 196-210 (2013).

[4] X. Zhang, A. Hosseini, H. Subbaraman, S. Wang, Q. Zhan, J. Luo, A. K. Jen, and R. T. Chen, "Integrated Photonic Electromagnetic Field Sensor Based on Broadband Bowtie Antenna Coupled Silicon Organic Hybrid Modulator," Lightwave Technology, Journal of, 32(20), 3774-3784 (2014).

[5] Y. Shi, C. Zhang, H. Zhang, J. H. Bechtel, L. R. Dalton, B. H. Robinson, and W. H. Steier, "Low (sub-1-volt) halfwave voltage polymeric electro-optic modulators achieved by controlling chromophore shape," Science, 288(5463), 119-122 (2000).

[6] D. Chen, H. R. Fetterman, A. Chen, W. H. Steier, L. R. Dalton, W. Wang, and Y. Shi, "Demonstration of 110 GHz electro-optic polymer modulators," Applied Physics Letters, 70(25), 3335-3337 (1997).

[7] R. T. Chen, L. Lin, C. Choi, Y. J. Liu, B. Bihari, L. Wu, S. Tang, R. Wickman, B. Picor, and M. Hibb-Brenner, "Fully embedded board-level guided-wave optoelectronic interconnects," Proceedings of the IEEE, 88(6), 780793 (2000).

[8] X. Lin, T. Ling, H. Subbaraman, X. Zhang, K. Byun, L. J. Guo, and R. T. Chen, "Ultraviolet imprinting and aligned ink-jet printing for multilayer patterning of electro-optic polymer modulators," Optics letters, 38(10), 1597-1599 (2013).

[9] G. T. Reed, G. Mashanovich, F. Gardes, and D. Thomson, "Silicon optical modulators," Nature Photonics, 4(8), 518-526 (2010).

[10] R. Soref, "The past, present, and future of silicon photonics," Selected Topics in Quantum Electronics, IEEE Journal of, 12(6), 1678-1687 (2006).

[11] T. Baba, "Slow light in photonic crystals," Nature Photonics, 2(8), 465-473 (2008).

[12] Y. A. Vlasov, M. O'Boyle, H. F. Hamann, and S. J. McNab, "Active control of slow light on a chip with photonic crystal waveguides," Nature, 438(7064), 65-69 (2005).

[13] Y. Jiang, W. Jiang, L. Gu, X. Chen, and R. T. Chen, "80-micron interaction length silicon photonic crystal waveguide modulator," Applied Physics Letters, 87(22), 221105 (2005).

[14] H. C. Nguyen, S. Hashimoto, M. Shinkawa, and T. Baba, "Compact and fast photonic crystal silicon optical modulators," Optics Express, 20(20), 22465-22474 (2012).

[15] C.-Y. Lin, X. Wang, S. Chakravarty, B. S. Lee, W. Lai, J. Luo, A. K.-Y. Jen, and R. T. Chen, "Electro-optic polymer infiltrated silicon photonic crystal slot waveguide modulator with $23 \mathrm{~dB}$ slow light enhancement," Applied Physics Letters, 97(9), 093304 (2010).

[16] S. Huang, T.-D. Kim, J. Luo, S. K. Hau, Z. Shi, X.-H. Zhou, H.-L. Yip, and A. K.-Y. Jen, "Highly efficient electro-optic polymers through improved poling using a thin TiO 2-modified transparent electrode," Applied Physics Letters, 96(24), 243311-243311-3 (2010).

[17] H. Chen, B. Chen, D. Huang, D. Jin, J. Luo, A.-Y. Jen, and R. Dinu, "Broadband electro-optic polymer modulators with high electro-optic activity and low poling induced optical loss," Applied Physics Letters, 93(4), 043507 (2008).

[18] T. Baehr-Jones, B. Penkov, J. Huang, P. Sullivan, J. Davies, J. Takayesu, J. Luo, T.-D. Kim, L. Dalton, and A. Jen, "Nonlinear polymer-clad silicon slot waveguide modulator with a half wave voltage of $0.25 \mathrm{~V}$," Applied 
Physics Letters, 92(16), 163303 (2008).

[19] H. C. Nguyen, Y. Sakai, M. Shinkawa, N. Ishikura, and T. Baba, "10 Gb/s operation of photonic crystal silicon optical modulators," Optics Express, 19(14), 13000-13007 (2011).

[20] X. Zhang, H. Subbaraman, A. Hosseini, and R. T. Chen, "Highly efficient mode converter for coupling light into wide slot photonic crystal waveguide," Optics Express, 22(17), 20678-20690 (2014).

[21] A. Hosseini, X. Xu, D. N. Kwong, H. Subbaraman, W. Jiang, and R. T. Chen, "On the role of evanescent modes and group index tapering in slow light photonic crystal waveguide coupling efficiency," Applied Physics Letters, 98(3), 031107-031107-3 (2011).

[22] X. Xu, H. Subbaraman, J. Covey, D. Kwong, A. Hosseini, and R. T. Chen, "Complementary metal-oxidesemiconductor compatible high efficiency subwavelength grating couplers for silicon integrated photonics," Applied Physics Letters, 101(3), 031109-031109-4 (2012).

[23] M. Soljačić, S. G. Johnson, S. Fan, M. Ibanescu, E. Ippen, and J. Joannopoulos, "Photonic-crystal slow-light enhancement of nonlinear phase sensitivity," JOSA B, 19(9), 2052-2059 (2002).

[24] J. Witzens, T. Baehr-Jones, and M. Hochberg, "Design of transmission line driven slot waveguide MachZehnder interferometers and application to analog optical links," Optics Express, 18(16), 16902-16928 (2010).

[25] J.-M. Brosi, C. Koos, L. C. Andreani, M. Waldow, J. Leuthold, and W. Freude, "High-speed low-voltage electrooptic modulator with a polymer-infiltrated silicon photonic crystal waveguide," Optics Express, 16(6), 41774191 (2008).

[26] J. Leuthold, C. Koos, W. Freude, L. Alloatti, R. Palmer, D. Korn, J. Pfeifle, M. Lauermann, R. Dinu, and S. Wehrli, "Silicon-organic hybrid electro-optical devices," Selected Topics in Quantum Electronics, IEEE Journal of, 19(6), 3401413-3401413 (2013).

[27] J. H. WŘlbern, A. Petrov, and M. Eich, "Electro-optical modulator in a polymerinfiltrated silicon slotted photonic crystal waveguide heterostructure resonator," Optics Express, 17(1), 304-313 (2009).

[28] X. Zhang, A. Hosseini, X. Xu, S. Wang, Q. Zhan, Y. Zou, S. Chakravarty, and R. T. Chen, "Electric field sensor based on electro-optic polymer refilled silicon slot photonic crystal waveguide coupled with bowtie antenna," in SPIE Photonic West 2013: Terahertz, RF, Millimeter, and Submillimeter-Wave Technology and Applications VI, 2013, p. 862418.

[29] S. K. Ghandhi, [VLSI fabrication principles: silicon and gallium arsenide] John Wiley \& Sons, (2008).

[30] J. Doylend, P. Jessop, and A. Knights, "Optical attenuation in ion-implanted silicon waveguide racetrack resonators," Opt. Express19 (16), 14913-14918 (2011).

[31] A. Chen, H. Sun, A. Szep, S. Shi, D. Prather, Z. Lin, R. S. Kim, and D. Abeysinghe, "Achieving higher modulation efficiency in electrooptic polymer modulator with slotted silicon waveguide," Lightwave Technology, Journal of, 29(21), 3310-3318 (2011).

[32] L. Alloatti, D. Korn, R. Palmer, D. Hillerkuss, J. Li, A. Barklund, R. Dinu, J. Wieland, M. Fournier, and J. Fedeli, "42.7 Gbit/s electro-optic modulator in silicon technology," Optics Express, 19(12), 11841-11851 (2011).

[33] R. Palmer, A. Luca, D. Korn, P. Schindler, M. Baier, J. Bolten, T. Wahlbrink, M. Waldow, R. Dinu, and W. Freude, "Low power mach-zehnder modulator in silicon-organic hybrid technology," Photonics Technology Letters, IEEE, 25(13), (2013).

[34] L. Alloatti, M. Lauermann, C. Sürgers, C. Koos, W. Freude, and J. Leuthold, "Optical absorption in silicon layers in the presence of charge inversion/accumulation or ion implantation," Applied Physics Letters, 103(5), 051104 (2013).

[35] C.-Y. Lin, A. X. Wang, B. S. Lee, X. Zhang, and R. T. Chen, "High dynamic range electric field sensor for electromagnetic pulse detection," Optics Express, 19, 17372-17377 (2011).

[36] X. Zhang, B. Lee, C.-y. Lin, A. X. Wang, A. Hosseini, and R. T. Chen, "Highly Linear Broadband Optical Modulator Based on Electro-Optic Polymer," Photonics Journal, IEEE, 4(6), 2214-2228 (2012).

[37] X. Zhang, A. Hosseini, J. Luo, A. K.-Y. Jen, and R. T. Chen, "Hybrid silicon-electro-optic-polymer integrated high-performance optical modulator," in SPIE Photonic West, OPTO, 2014, pp. 89910O-89910O-6.

[38] X. Zhang, A. Hosseini, S. Chakravarty, J. Luo, A. K.-Y. Jen, and R. T. Chen, "Wide optical spectrum range, subvolt, compact modulator based on an electro-optic polymer refilled silicon slot photonic crystal waveguide," Optics letters, 38(22), 4931-4934 (2013).

[39] A. Hosseini, X. Xu, H. Subbaraman, C.-Y. Lin, S. Rahimi, and R. T. Chen, "Large optical spectral range dispersion engineered silicon-based photonic crystal waveguide modulator," Opt. Express20 (11), 12318-12325 (2012).

[40] T. F. Krauss, "Slow light in photonic crystal waveguides," Journal of Physics D: Applied Physics, 40(9), 2666 (2007). 
[41] S.-i. Inoue, and A. Otomo, "Electro-optic polymer/silicon hybrid slow light modulator based on one-dimensional photonic crystal waveguides," Applied Physics Letters, 103(17), 171101 (2013).

[42] J. H. Wülbern, S. Prorok, J. Hampe, A. Petrov, M. Eich, J. Luo, A. K.-Y. Jen, M. Jenett, and A. Jacob, “40 GHz electro-optic modulation in hybrid silicon-organic slotted photonic crystal waveguides," Optics letters, 35(16), 2753-2755 (2010).

[43] L. D. Tzuang, M. Soltani, Y. H. D. Lee, and M. Lipson, "High RF carrier frequency modulation in silicon resonators by coupling adjacent free-spectral-range modes," Optics letters, 39(7), 1799-1802 (2014).

[44] Y. N. Wijayanto, H. Murata, and Y. Okamura, "Electro-optic microwave-lightwave converters utilizing patch antennas with orthogonal gaps," Journal of Nonlinear Optical Physics \& Materials, 21(01), (2012).

[45] A. Melikyan, L. Alloatti, A. Muslija, D. Hillerkuss, P. Schindler, J. Li, R. Palmer, D. Korn, S. Muehlbrandt, and D. Van Thourhout, "High-speed plasmonic phase modulators," Nature Photonics, (2014).

[46] O. Herrera, K. Kim, R. Voorakaranam, R. Himmelhuber, S. Wang, Q. Zhan, L. Li, R. Norwood, R. Neilson, and J. Luo, "Silica/Electro-optic Polymer Optical Modulator with Integrated Antenna for Microwave Receiving."

[47] H. Bartzsch, D. Glöß, P. Frach, M. Gittner, E. Schultheiß, W. Brode, and J. Hartung, "Electrical insulation properties of sputter - deposited $\mathrm{SiO} 2$, Si3N4 and A12O3 films at room temperature and $400^{\circ} \mathrm{C}$," Physica Status Solidi (A), 206(3), 514-519 (2009).

[48] S. M. Sze, and K. K. Ng, [Physics of semiconductor devices] John Wiley \& Sons, (2006).

[49] D. A. Miller, "Energy consumption in optical modulators for interconnects," Optics Express, 20(102), A293A308 (2012).

[50] J. Leuthold, C. Koos, W. Freude, L. Alloatti, R. Palmer, D. Korn, J. Pfeifle, M. Lauermann, R. Dinu, and S. Wehrli, "High-speed, low-power optical modulators in silicon," in Transparent Optical Networks (ICTON), 2013 15th International Conference on, 2013, pp. 1-4.

[51] R. Palmer, S. Koeber, W. Heni, D. Elder, D. Korn, H. Yu, L. Alloatti, S. Koenig, P. Schindler, and W. Bogaerts, "High-speed silicon-organic hybrid (SOH) modulator with 1, $6 \mathrm{fJ} / \mathrm{bit}$ and $180 \mathrm{pm} / \mathrm{V}$ in-device nonlinearity," in 39th European Conference and Exhibition on Optical Communication (ECOC-2013), 2013

[52] H. C. Nguyen, Y. Sakai, M. Shinkawa, N. Ishikura, and T. Baba, "Photonic crystal silicon optical modulators: carrier-injection and depletion at $10 \mathrm{~Gb} / \mathrm{s}$," Quantum Electronics, IEEE Journal of, 48(2), 210-220 (2012).

[53] A. Hosseini, X. Xu, H. Subbaraman, C.-Y. Lin, S. Rahimi, and R. T. Chen, "Large optical spectral range dispersion engineered silicon-based photonic crystal waveguide modulator," Opt. Express, 20(11), 12318-12325 (2012).

[54] M. Gould, T. Baehr-Jones, R. Ding, S. Huang, J. Luo, A. K.-Y. Jen, J.-M. Fedeli, M. Fournier, and M. Hochberg, "Silicon-polymer hybrid slot waveguide ring-resonator modulator," Optics Express, 19(5), 3952-3961 (2011).

[55] C. Zhang, T. Ling, S.-L. Chen, and L. J. Guo, "Ultrabroad Bandwidth and Highly Sensitive Optical Ultrasonic Detector for Photoacoustic Imaging," ACS Photonics, (2014).

[56] L. Chen, Q. Xu, M. G. Wood, and R. M. Reano, "Hybrid silicon and lithium niobate electro-optical ring modulator," Optica, 1(2), 112-118 (2014).

[57] C. Xiong, W. H. Pernice, J. H. Ngai, J. W. Reiner, D. Kumah, F. J. Walker, C. H. Ahn, and H. X. Tang, “Active silicon integrated nanophotonics: ferroelectric BaTiO3 devices," Nano letters, 14(3), 1419-1425 (2014).

[58] Y. Terada, and T. Baba, "Low-Voltage 25 Gbps Modulators Based On Si Photonic Crystal Slow Light Waveguides," in CLEO: Science and Innovations, 2014, p. SM2G. 2.

[59] L. Alloatti, R. Palmer, S. Diebold, K. P. Pahl, B. Q. Chen, R. Dinu, M. Fournier, J. M. Fedeli, T. Zwick, W. Freude, C. Koos, and J. Leuthold, "100 GHz silicon-organic hybrid modulator," Light-Science \& Applications, 3, (2014).

[60] R. Palmer, S. Koeber, D. L. Elder, M. Woessner, W. Heni, D. Korn, M. Lauermann, W. Bogaerts, L. Dalton, and W. Freude, "High-Speed, Low Drive-Voltage Silicon-Organic Hybrid Modulator Based on a BinaryChromophore Electro-Optic Material," Journal of Lightwave Technology, 32(16), 2726-2734 (2014).

[61] S. Huang, J. Luo, H. L. Yip, A. Ayazi, X. H. Zhou, M. Gould, A. Chen, T. Baehr - Jones, M. Hochberg, and A. K. Y. Jen, "Efficient Poling of Electro - Optic Polymers in Thin Films and Silicon Slot Waveguides by Detachable Pyroelectric Crystals,” Advanced Materials, 24(10), OP42-OP47 (2012).

[62] D. Korn, R. Palmer, H. Yu, P. C. Schindler, L. Alloatti, M. Baier, R. Schmogrow, W. Bogaerts, S. K. Selvaraja, and G. Lepage, "Silicon-organic hybrid (SOH) IQ modulator using the linear electro-optic effect for transmitting 16QAM at $112 \mathrm{Gbit} / \mathrm{s}, "$ Optics Express, 21(11), 13219-13227 (2013).

[63] C. Weimann, P. Schindler, R. Palmer, S. Wolf, D. Bekele, D. Korn, J. Pfeifle, S. Koeber, R. Schmogrow, and L. Alloatti, "Silicon-organic hybrid (SOH) frequency comb sources for terabit/s data transmission," Optics Express, 22(3), 3629-3637 (2014). 
[64] M. Lauermann, R. Palmer, S. Koeber, P. C. Schindler, D. Korn, T. Wahlbrink, J. Bolten, M. Waldow, D. L. Elder, L. R. Dalton, J. Leuthold, W. Freude, and C. Koos, "Low-power silicon-organic hybrid (SOH) modulators for advanced modulation formats," Optics Express, 22(24), 29927-29936 (2014).

[65] S. Takahashi, B. Bhola, A. Yick, W. Steier, J. Luo, A. Y. Jen, D. Jin, and R. Dinu, "Photo-Stability Measurement of Electro-Optic Polymer Waveguides With High Intensity at 1550-nm Wavelength,” Journal of Lightwave Technology, 27(8), 1045-1050 (2009).

[66] S. Huang, J. Luo, Z. Jin, X.-H. Zhou, Z. Shi, and A. K.-Y. Jen, "Enhanced temporal stability of a highly efficient guest-host electro-optic polymer through a barrier layer assisted poling process," Journal of Materials Chemistry, 22(38), 20353-20357 (2012).

[67] Z. Shi, J. Luo, S. Huang, B. M. Polishak, X.-H. Zhou, S. Liff, T. R. Younkin, B. A. Block, and A. K.-Y. Jen, "Achieving excellent electro-optic activity and thermal stability in poled polymers through an expeditious crosslinking process," Journal of Materials Chemistry, 22(3), 951-959 (2012). 